\title{
One-loop effective potential on hyperbolic manifolds
}

\author{
Guido Cognola*, Klaus Kirsten and Sergio Zerbini* \\ Dipartimento di Fisica, Università di Trento, Italia ${ }^{\dagger}$
}

February 1993

U.T.F. 284

Abstract: The one-loop effective potential for a scalar field defined on an ultrastatic space-time whose spatial part is a compact hyperbolic manifold, is studied using zeta-function regularization for the one-loop effective action. Other possible regularizations are discussed in detail. The renormalization group equations are derived and their connection with the conformal anomaly is pointed out. The symmetry breaking and the topological mass generation are also discussed.

PACS numbers: $03.70 .+k$ Theory of quantized fields

11.10.Gh Renormalization

04.90.+e Other topics in relativity and gravitation

\section{Introduction}

In the last decades, there has been a great deal of investigations on the properties of interacting quantum field theories in curved space-time (see for example the text book [1]

${ }^{*}$ Istituto Nazionale di Fisica Nucleare, Gruppo Collegato di Trento, Italia

†Email: cognola@itncisca.bitnet, kirsten@itncisca.bitnet, zerbini@itncisca.bitnet 
and references cited therein). More recently the inflationary models have been proposed in order to overcome some difficulties present in the standard cosmological model (see for example [2] and references therein). Several techniques have been employed, among these the background-field method within path-integral approach to quantum field theory [3, 4], which is very useful in dealing with the one-loop approximation. In addition there have been attemps to investigate the role of the topology and the phenomenon of the topological mass generation has been discovered [5, 6, 7, 8]. Furthermore "topological symmetry restoration" [9, 10, 11] and later "topological symmetry breaking" [12, 13 have been investigated in the self-interacting scalar theory. The non trivial topological space-times which mainly have been considered are the ones with the torus topology, with the exception of ref. [11], in which untwisted scalar fields on $S^{3} / \Gamma$ are discussed $(\Gamma$ is a discrete group of isometries of $S^{3}$ ). Very recently, such manifolds have been considered also by Dowker et al. 14.

In this paper we shall consider a self-interacting scalar field defined on an ultrastatic space-time in which the spatial section is a compact hyperbolic 3-dimensional manifold, namely it can be represented as $H^{3} / \Gamma, \Gamma$ being a discrete group of isometries of $H^{3}$, the Lobachevsky space. It is well known that such space-times have a highly non trivial topology and, for a fixed value of cosmological time, they are spatially homogeneous isotropic universe models with negative constant curvature. It is reasonable to assume that such space-times have some physical relevance. In fact, as it has been stressed in ref. [15], they would affect the occurence of the particle horizons and in principle they could be tested experimentally by means of observation. There is also a mathematical motivation based on the Thurston conjecture [16] which, roughly speaking, states that every 3-dimensional compact manifold admits a canonical decomposition into eight primitive pieces. Among 
such primitive pieces, the hyperbolic one is the most important, the other contributions being exceptional cases. As a consequence, if one is interested in studying matter fields defined on space-times with a compact spatial section, the compact hyperbolic case might play an important physical role. In fact one of the results achieved in this paper is an expression for the square of the topological mass. The sign of this depends on the characters of the fields. For trivial character (untwisted fields) the sign is positive (this might help to restore symmetry) whereas for twisted fields there could exist a mechanism for symmetry breaking.

The plan of the paper is the following. In Section 2, the scalar model is introduced and the definition of the regularized one-loop effective potential within the background field method is summarized. It is also shown that the finite part of the one-loop effective potential in $R^{D}$ can be determined without the explicit knowledge of the regularization functions. In Section 3, making use of the Selberg trace formula, the one-loop effective potential for $R \times H^{3} / \Gamma$ is computed. In Section 4 , the renormalization program is carried out. In Section 5 a derivation of the renormalization group equations at one-loop level is presented for the sake of completeness, paying attention to the role of the conformal anomaly. In Section 6, symmetry breaking and topological mass generation are discussed. The conclusions are summarized in Section 7. In Appendix A, several regularizations are discussed in order to illustrate the general result obtained in Section 2. Finally, Appendix B contains some material on heat kernel expansion and zeta-function regularization. 


\section{One loop-effective potential}

Before we restrict our attention to the hyperbolic manifold $R \times H^{3} / \Gamma$, let us deal with a scalar field $\phi$ defined on an arbitrary smooth D-dimensional manifold $\mathcal{M}$ (Euclidean signature), for simplicity without boundary. To begin with, we recall that the Euclidean generating functional reads

$$
W_{E}[J]=N_{E} \int d[\phi] e^{-S_{E}[\phi, J]}
$$

where

$$
\begin{aligned}
& S_{E}[\phi, J]=\int_{\mathcal{M}}\left[\frac{1}{2} \phi L \phi+V_{o}(\phi)-J \phi\right] d v o l \\
& L=-\triangle_{D}+m^{2}+\xi R
\end{aligned}
$$

Here $\triangle_{D}$ is the Laplace-Beltrami operator in $\mathcal{M}, R$ is the scalar curvature, $\xi$ an arbitrary numerical parameter and finally $V_{o}(\phi)$ is the potential describing the self-interaction of the field.

If we denote the classical solution by $\phi_{c}$, the one loop effective action takes the form

$$
\begin{aligned}
\Gamma\left[\phi_{c}\right] & =S_{E}\left[\phi_{c}\right]+\frac{1}{2} \ln \operatorname{det} \frac{A}{\mu^{2}}=S_{E}\left[\phi_{c}\right]+\frac{1}{2} \ln \operatorname{det} \frac{L+V_{o}^{\prime \prime}\left(\phi_{c}\right)}{\mu^{2}} \\
& =\int\left[V\left(\phi_{c}\right)+\frac{1}{2} Z\left(\phi_{c}\right) g^{i j} \partial_{i} \phi_{c} \partial_{j} \phi_{c}+\cdots\right] d v o l
\end{aligned}
$$

in which $A$ is a positive definite elliptic operator of second order defined on $\mathcal{M}$ (the small disturbance or fluctuation operator) and $\mu$ is an arbitrary normalization parameter coming from the scalar path-integral measure and necessary in order to keep the zetafunction dimensionless for all $s$. The latter equation defines the one-loop effective potential $V\left(\phi_{c}\right)$. Of course it is equal to the classical one $V^{(0)}\left(\phi_{c}\right)=V_{o}\left(\phi_{c}\right)+\left(m^{2}+\xi R\right) \phi_{c}^{2} / 2$, plus 
quantum corrections $V^{(1)}\left(\phi_{c}\right)$ of order $\hbar$. The quantum corrections to the classical action are formally divergent, because the eigenvalues of $A$ increase without bound. One has to use a suitable regularization procedure.

In the following we shall employ a class of regularizations based on the Schwinger representation (see for example [17]). In order to work with dimensionless quantities, we put $B=\mu^{-2} A$. The regularized determinant of the operator $B$ is defined by

$$
(\ln \operatorname{det} B)_{\varepsilon}=-\int_{0}^{\infty} d t t^{-1} \varrho(\varepsilon, t) \operatorname{Tr} e^{-t B}
$$

where $\varrho(\varepsilon, t)$ is a suitable regularization function of the dimensionless parameter $t$, which has to satisfy the two requirements we are going to discuss. First, for fixed $t>0$, the limit as $\varepsilon$ goes to zero must be equal to one. Second, for fixed and sufficiently large $\varepsilon$, $\varrho(\varepsilon, t)$ has to regularize the singularity at $t=0$ coming from the heat kernel expansion

$$
\operatorname{Tr} e^{-t B} \sim \sum_{n} A_{n} t^{n-D / 2}
$$

$A_{n}$ being the integrated Seeley-DeWitt coefficients associated with the operator $B$. The analytic continuation will be used to reach small values of $\varepsilon$. These requirements do not uniquely determine the regularization function $\varrho$. In fact, in the following we shall examine several cases.

Using eq. (2.6) in eq. (2.5) one can easily see that the number of divergent terms for $\varepsilon \rightarrow 0$ is equal to $Q+1, Q$ being the integer part of $D / 2$. They are proportional to the Seeley-De Witt coefficients $A_{0}, \ldots A_{Q}$ (which contain the full dependence on the geometry), the prefactors depending on the regularization function [17]. In the Appendix A, this general result is verified explicitly in several examples.

In the rest of this Section, in the particular but important case of $R^{D}$, we would like to show that the finite part of the effective potential is uniquely determined, modulo a 
constant which can be absorbed by the arbitrary scale parameter $\mu$, and can be evaluated without making use of the explicit knowledge of the regularization functions . As usual, in $R^{D}$ we consider a large region of volume $V(\mathcal{M})$ and the limit $V(\mathcal{M}) \rightarrow \infty$ shall be taken at the end of the calculations. As we will see in the following section, apart from a topological contribution which will be obtained using the Selberg trace formula, this calculation is already enough to give the one loop-effective potential in the constant curvature space-time $R \times H^{3} / \Gamma$ under consideration.

By definition, the effective potential has to be evaluated by considering a constant $V_{o}^{\prime \prime}$. This means that

$$
\frac{\operatorname{Tr} e^{-t B}}{V(\mathcal{M})}=\frac{\mu^{D} e^{-t f^{2} / \mu^{2}}}{(4 \pi t)^{D / 2}}
$$

where $f^{2}=m^{2}+V_{o}^{\prime \prime}\left(\phi_{c}\right)$ is a positive constant. As a consequence, from eqs. (2.4) and (2.5), we obtain the regularized expression $V(\varepsilon, f)$ for $V^{(1)}\left(\phi_{c}\right)$,

$$
V(\varepsilon, f)=\frac{(\ln \operatorname{det} B)_{\varepsilon}}{2 V(\mathcal{M})}=-\frac{1}{2}\left(\frac{\mu^{2}}{4 \pi}\right)^{D / 2} \int_{0}^{\infty} d t t^{-(1+D / 2)} \varrho(\varepsilon, t) e^{-t f^{2} / \mu^{2}}
$$

Now it is convenient to distinguish between even $(D=2 Q)$ and odd $(D=2 Q+1)$ dimensions. In order to derive a more explicit form of the divergent terms for $\varepsilon \rightarrow 0$, let us consider the $Q^{t h}$-derivative of $V(\varepsilon)$, which is

$$
\frac{d^{Q} V(\varepsilon, f)}{d y^{Q}}=\frac{(-1)^{Q}}{2}\left(\frac{\mu^{2}}{4 \pi}\right)^{D / 2} B_{e, o}(\varepsilon, y)
$$

where

$$
\begin{aligned}
& B_{e}(\varepsilon, y)=-\int_{0}^{\infty} d t t^{-1} \varrho(\varepsilon, t) e^{-t y}=\ln y+b+c_{Q}(\varepsilon)+\mathcal{O}(\varepsilon) \\
& B_{o}(\varepsilon, y)=-\int_{0}^{\infty} d t t^{-3 / 2} \varrho(\varepsilon, t) e^{-t y}=2 \sqrt{\pi y}+b+c_{Q}(\varepsilon)+\mathcal{O}(\varepsilon)
\end{aligned}
$$


In the derivation of the above equations we have made use of the properties of the regularization functions. Furthermore, in eqs. (2.10) and (2.11) $b$ is a constant and $c_{Q}(\varepsilon)$ is a function of $\varepsilon$, but not of $y$. We have used the same symbols for even and odd dimensions, but of course they represent different quantities in the two cases.

Making use of eqs. (2.10) and (2.11) in eq. (2.9) a simple integration gives

$$
\begin{aligned}
& V_{2 Q}(\varepsilon, f)=\frac{(-1)^{Q}}{2 Q !(4 \pi)^{Q}}\left[\ln \frac{f^{2}}{\mu^{2}}-C_{Q}+b\right] f^{D}+\mu^{D} \sum_{n=0}^{Q} c_{n}(\varepsilon)\left(\frac{f}{\mu}\right)^{2 n}+\mathcal{O}(\varepsilon) \\
& V_{2 Q+1}(\varepsilon, f)=\frac{(-1)^{Q} \Gamma(-Q-1 / 2)}{2(4 \pi)^{Q+1 / 2}} f^{D}+\mu^{D} \sum_{n=0}^{Q} c_{n}(\varepsilon)\left(\frac{f}{\mu}\right)^{2 n}+\mathcal{O}(\varepsilon)
\end{aligned}
$$

where we have set $C_{Q}=\sum_{n=1}^{Q} \frac{1}{n}$.

The dimensionless integration constants $c_{n}(\varepsilon)$, which are divergent for $\varepsilon \rightarrow 0$, define the counterterms which must be introduced in order to remove the divergences. For the particularly interesting case $D=4$ one gets

$$
V(\varepsilon, f)=\frac{f^{4}}{64 \pi^{2}}\left[\ln \frac{f^{2}}{\mu^{2}}-\frac{3}{2}+b\right]+c_{2}(\varepsilon) f^{4}+c_{1}(\varepsilon) \mu^{2} f^{2}+c_{0}(\varepsilon) \mu^{4}
$$

in agreement with the well known result obtained in refs. [18, 19], where some specific regularizations were used for $D=4$.

Some remarks are in order. The constants $b$ and $c_{n}(\varepsilon)$ depend on the choice of the regularization function, but $b$ can be absorbed by the arbitrary scale parameter $\mu$. As a result, the finite part of the effective potential does not depend on the regularization, as expected. In the Appendix A, several examples of admissible regularizations are reported as an illustration of the above general result. 


\section{The effective potential on $R \times H^{3} / \Gamma$}

In the following we shall consider the scalar field defined on a topologically non trivial space-time $R \times H^{D-1} / \Gamma$, that is a space-time with a hyperbolic $N=D-1$ dimensional manifold as a compact spatial part and with constant scalar curvature $R=N(N-1) \kappa$. Note that $R$ is also the scalar curvature of $H^{N}$. We shall explicitly compute the effective potential only for the physical interesting case $N=3$, but the extension to any $N$ is possible.

The manifold $H^{N} / \Gamma$ can be regarded as a quotient of the Lobachevsky space $H^{N}$ by a fixed-point free discontinuous group $\Gamma$ of isometries. The $\Gamma$ is assumed to be torsion-free. As usual, for the moment we will consider the case normalized to $\kappa=-1$, so until not otherwise stated all quantities are dimensionless. The results for arbitrary $\kappa$ are simply found by dimensional reasoning because $H^{N} / \Gamma$ is a homogeneous manifold.

If $h(r)$ is an even and holomorphic function in a strip of width $N$ about the real axis, and if $h(r)=\mathcal{O}\left(r^{-(N+1+\varepsilon)}\right)$ uniformly in this strip as $r \rightarrow \infty$, then the Selberg trace formula holds (see for example refs. 20, 21])

$$
\sum_{j=0}^{\infty} h\left(r_{j}\right)=V(\mathcal{F}) \int_{0}^{\infty} d r h(r) \Phi_{N}(r)+\sum_{\wp} \sum_{n=1}^{\infty} \frac{\chi\left(P^{n}(\gamma)\right)}{S_{N}\left(n ; l_{\gamma}\right)} \hat{h}\left(n l_{\gamma}\right)
$$

Here $V(\mathcal{F})$ is the volume of the fundamental domain $\mathcal{F}, \hat{h}$ is the Fourier transform of $h$, $\gamma \in \Gamma$ is an element of the conjugacy class associated with the length of a closed geodesic $l_{\gamma}, \wp$ is a set of primitive closed geodesics on the compact manifold and each $\gamma \in \wp$ determines the holonomy element $P(\gamma)$. Moreover, $S_{N}\left(n ; l_{\gamma}\right)$ is a known function of the conjugacy class (see ref. [22 for details) and $\chi(P)$ represents the characters, which define all topological inequivalent scalar fields. The sum on the left hand side of eq. (3.1) is over 
all positive $r_{j}$ satisfying

$$
r_{j}^{2}+\rho_{N}^{2}=-\lambda_{j}
$$

$\lambda_{j}$ being the eigenvalues of the automorphic Laplacian $\triangle_{N}(\Gamma, \chi)$ for a group $\Gamma$ and representation $\chi$, and $\rho_{N}=(N-1) / 2$.

The quantity $\Phi_{N}(r)$ is related to the Harish-Chandra function and reads

$$
\Phi_{N}(r)=\frac{2}{(4 \pi)^{N / 2} \Gamma(N / 2)}\left|\frac{\Gamma\left(\rho_{N}+i r\right)}{\Gamma(i r)}\right|^{2}
$$

In particular we have

$$
\Phi_{2}(r)=\pi r \tanh \pi r ; \quad \Phi_{3}(r)=\frac{r^{2}}{2 \pi^{2}}
$$

and the recurrence relation

$$
\Phi_{N+2}=\frac{1}{2 \pi N}\left[\rho_{N}^{2}+r^{2}\right] \Phi_{N}(r)
$$

which permits to obtain any $\Phi_{N}$ starting from $\Phi_{2}$ or $\Phi_{3}$ according to whether $N$ is even or odd.

We are interested in

$$
V(\varepsilon)=-\frac{1}{2 V(\mathcal{M})} \int_{0}^{\infty} d t t^{-1} \varrho(\varepsilon, t) \operatorname{Tr} e^{-t B_{D}}=-\frac{\mu}{2 V(\mathcal{F})} \int_{0}^{\infty} d t t^{-1} \varrho(\varepsilon, t) \frac{\operatorname{Tr} e^{-t B_{N}}}{(4 \pi t)^{1 / 2}}(3
$$

where $B_{N}=\mu^{-2}\left\{-\triangle_{N}+m^{2}+\xi R+V_{o}^{\prime \prime}\left(\phi_{c}\right)\right\}$, so we choose $h(r)=e^{-t \mu^{-2}\left(r^{2}+a^{2}\right)}$, with

$$
a^{2}=\rho_{N}^{2}+m^{2}+\xi R+V_{o}^{\prime \prime}\left(\phi_{c}\right)
$$

and evaluate $\operatorname{Tr} \exp \left(-t B_{N}\right)$ by using the Selberg trace formula (3.1).

For $N=3$ we obtain

$$
\operatorname{Tr} e^{-t B_{3}}=\frac{V(\mathcal{F}) \mu^{3} e^{-t a^{2} / \mu^{2}}}{(4 \pi t)^{3 / 2}}+F_{3}(t)
$$


where $F_{3}(t)$ is the contribution due to topology. It can be easily computed for any $N$ and takes the form

$$
F_{N}(t)=\frac{\mu e^{-t a^{2} / \mu^{2}}}{(4 \pi t)^{1 / 2}} \sum_{\wp} \sum_{n=1}^{\infty} \frac{\chi\left(P^{n}(\gamma)\right)}{S_{N}\left(n ; l_{\gamma}\right)} e^{-\left(n l_{\gamma} \mu\right)^{2} / 4 t}
$$

As mentioned in Section 2, in eq. (3.8) the contribution due to the identity element of the group $\Gamma$ is formally equivalent to the one we have already considered in the flat case. The only difference is that $f$ is replaced by $a$. It has to be noted that this is a peculiarity of 3 dimensions. When $N=2 Q+1>3$, the contribution due to the identity element of the group $\Gamma$ is a polynomial in the variable $t^{-1 / 2}$, multiplied by the exponential factor $\exp \left(-t a^{2} / \mu^{2}\right)$. It contains $(N-1) / 2$ terms proportional to $t^{-3 / 2} \ldots t^{-N / 2}$. Of course, the leading term is formally equivalent to the flat one. The situation is more complicated for even $N$. In this case in fact, the identity element of the group $\Gamma$ produces, together with a polynomial of order $N / 2$ in the variable $t^{-1}$, multiplied by the exponential factor $\exp \left(-t a^{2} / \mu^{2}\right)$, also a complicated regular function for $t \rightarrow 0$.

The function $F_{3}(t)$ gives a contribution to the one-loop effective potential, which we refer to as the topological one. It can be represented in terms of the Selberg zeta function $Z(s)$, which may be defined by means of the equation

$$
\frac{d}{d s} \ln Z(s)=\sum_{\wp} \sum_{n=1}^{\infty} \frac{\chi\left(P^{n}(\gamma)\right)}{S_{N}\left(n ; l_{\gamma}\right)} e^{-\left(s-\rho_{N}\right) n l_{\gamma}}
$$

It has to be noted that in the evaluation of the topological contribution $V_{T}$ to the effective potential, we can put the regularization function $\varrho(\varepsilon, t)$ equal to 1 since all the ultraviolet divergences come from the identity part. This means that the counterterms are not affected by the presence of the non trivial topology [23, 24, 25]. In order to analyze the dependence of the final result on the curvature, let us now introduce arbitrary constant 
curvature $R$. Then we have $l_{\gamma} \sim 1 / \sqrt{|\kappa|}, V(\mathcal{F}) \sim|\kappa|^{-\frac{3}{2}}$, furthermore $a^{2}=m^{2}+(\xi-$ $1 / 6) R+V_{o}^{\prime \prime}\left(\phi_{c}\right)$, and we find $V(\varepsilon)=V(\varepsilon, a)+V_{T}, V(\varepsilon, a)$ being given in eq. (2.12) with $Q=2$ and

$$
V_{T}=-\frac{a^{2}|\kappa|^{-1 / 2}}{2 \pi V(\mathcal{F})} \sum_{\wp} \sum_{n=1}^{\infty} \frac{\chi\left(P^{n}(\gamma)\right)}{S_{3}\left(n ; l_{\gamma}\right)} \frac{\mathbf{K}_{1}\left(n l_{\gamma} a\right)}{n l_{\gamma} a}
$$

From the latter equation we can see that for $a>0, V_{T}$ is exponentially vanishing when $\kappa$ goes to zero due to the fact that $l_{\gamma}$ diverges as $|\kappa|^{-1 / 2}$ when $\kappa \rightarrow 0$.

Making use of a suitable integral representation for $\mathbf{K}_{1}$ [26] and definition (3.10) we may write $V_{T}$ in the final form

$$
V_{T}=-\frac{a^{2}|\kappa|^{-1 / 2}}{2 \pi V(\mathcal{F})} \int_{1}^{\infty} d u \sqrt{u^{2}-1} \frac{Z^{\prime}}{Z}\left(1+u a|\kappa|^{-1 / 2}\right)
$$

in agreement with the zeta-function calculation [27.

\section{Renormalization of $\lambda \phi^{4}$ on $R \times H^{3} / \Gamma$}

In the previous section we have derived the regularized one-loop effective potential $V\left(\phi_{c}, R\right)=$ $V^{(0)}\left(\phi_{c}, R\right)+V^{(1)}\left(\phi_{c}, R\right)$ for a scalar field defined on the manifold $R \times H^{3} / \Gamma$. We now introduce $R$ into the notation to remind the reader of the nonvanishing curvature. The one-loop effective potential depends on the arbitrary parameter $\mu$, this dependence being eliminated by the renormalization procedure. To this aim, here we consider a scalar field self-interacting by a term of the kind $V_{o}(\phi)=\lambda \phi^{4} / 24$ and consider the classical potential

$$
\left.V_{c}\left(\phi_{c}, R\right)\right)=\Lambda+\frac{\lambda \phi_{c}^{4}}{24}+\frac{m^{2} \phi_{c}^{2}}{2}+\frac{\xi R \phi_{c}^{2}}{2}+\varepsilon_{0} R+\frac{\varepsilon_{1} R^{2}}{2}
$$

$\Lambda$ being the cosmological constant. Note that one is forced to take into consideration the most general quadratic gravitational Lagrangian, because the renormalization procedure gives rise in a natural manner to all such terms in the curvature [28]. 
We write the renormalized one-loop effective potential in the form

$$
V_{r}\left(\phi_{c}, R\right)=V_{c}\left(\phi_{c}, R\right)+V_{r}^{(1)}\left(\phi_{c}, R\right)
$$

where

$$
\left.V_{r}^{(1)}\left(\phi_{c}, R\right)\right)=\frac{a^{4}}{64 \pi^{2}}\left[\ln \frac{a^{2}}{\mu^{2}}-\frac{3}{2}\right]+V_{T}\left(\phi_{c}, R\right)+\delta V\left(\phi_{c}, R\right)
$$

with the counterterm contribution

$$
\delta V\left(\phi_{c}, R\right)=\delta \Lambda+\frac{\delta \lambda \phi_{c}^{4}}{24}+\frac{\delta m^{2} \phi_{c}^{2}}{2}+\frac{\delta \xi R \phi_{c}^{2}}{2}+\delta \varepsilon_{0} R+\frac{\delta \varepsilon_{1} R^{2}}{2}
$$

reflecting the structure of the divergences.

For convenience now we indicate by $\alpha=\alpha_{q}(q=0, \ldots, 5)$ the collection of the coupling constants, that is $\left(\alpha_{0}, \ldots, \alpha_{5}\right) \equiv\left(\Lambda, \lambda, m^{2}, \xi, \varepsilon_{0}, \varepsilon_{1}\right)$. The quantities $\delta \alpha$, which renormalize the coupling constants, are determined by the following renormalization conditions [29, 30]

$$
\begin{array}{ll}
\Lambda=\left.V_{r}\right|_{\phi_{c}=\varphi_{0}, R=0} ; \quad \lambda=\left.\frac{\partial^{4} V_{r}}{\partial \phi_{c}^{4}}\right|_{\phi_{c}=\varphi_{1}, R=R_{1}} ; & m^{2}=\left.\frac{\partial^{2} V_{r}}{\partial \phi_{c}^{2}}\right|_{\phi_{c}=0, R=0} ; \\
\xi=\left.\frac{\partial^{3} V_{r}}{\partial R \partial \phi_{c}^{2}}\right|_{\phi_{c}=\varphi_{3}, R=R_{3}} ; \quad \varepsilon_{0}=\left.\frac{\partial V_{r}}{\partial R}\right|_{\phi_{c}=0, R=0} ; \quad \varepsilon_{1}=\left.\frac{\partial^{2} V_{r}}{\partial R^{2}}\right|_{\phi_{c}=\varphi_{5}, R=R_{5}}
\end{array}
$$

By $\varphi_{0}$ we indicate the value of the field for which the potential has a true minimum. For $m^{2}+\xi R>0\left(\right.$ respectively $\left.m^{2}+\xi R<0\right)$ this value is zero (respectively $\left.\pm\left(-6\left[m^{2}+\xi R\right] / \lambda\right)^{\frac{1}{2}}\right)$ for the classical potential, but this may change for the one-loop effective one. The choice of different values $\phi_{c}=\varphi_{q}$ and $R=R_{q}$ in order to define the physical coupling constants is due to the fact that in general they are measured at different scales, the behaviour with respect to a change of scale being determined by the renormalization group eqs. (5.9), which we shall derive in the next Section. It has to be noted that by a suitable choice of $\varphi_{q}$ and $R_{q}$, equations (4.5) reduce to the conditions chosen by other authors (see for example refs. 29] and 30). 
By imposing conditions (4.5) we obtain the counterterms (we used an algebraic computing program)

$$
\begin{aligned}
\delta \Lambda= & -\frac{m^{2} \varphi_{0}^{2}}{2}-\frac{\lambda \varphi_{0}^{4}}{24}+\frac{\partial^{4} V_{T}\left(\varphi_{1}, R_{1}\right)}{\partial \phi_{c}^{4}} \frac{\varphi_{0}^{4}}{24}+\frac{1}{64 \pi^{2}}\left[M_{0}^{4}\left(\frac{3}{2}-\ln \frac{M_{0}^{2}}{\mu^{2}}\right)\right. \\
& \left.-\lambda m^{2} \varphi_{0}^{2}\left(1-\ln \frac{m^{2}}{\mu^{2}}\right)+\frac{\lambda^{2} \varphi_{0}^{4}}{12}\left(3 \ln \frac{M_{1}^{2}}{\mu^{2}}+\frac{6 \lambda \varphi_{1}^{2}}{M_{1}^{2}}-\frac{\lambda^{2} \varphi_{1}^{4}}{M_{1}^{4}}\right)\right] \\
\delta \lambda= & -\frac{\lambda^{2}}{32 \pi^{2}}\left[3 \ln \frac{M_{1}^{2}}{\mu^{2}}+\frac{6 \lambda \varphi_{1}^{2}}{M_{1}^{2}}-\frac{\lambda^{2} \varphi_{1}^{4}}{M_{1}^{4}}\right]-\frac{\partial^{4} V_{T}\left(\varphi_{1}, R_{1}\right)}{\partial \phi_{c}^{4}} \\
\delta m^{2}= & \frac{\lambda m^{2}}{32 \pi^{2}}\left[1-\ln \frac{m^{2}}{\mu^{2}}\right] \\
\delta \xi= & -\frac{\lambda(\xi-1 / 6)}{32 \pi^{2}}\left[\ln \frac{M_{3}^{2}}{\mu^{2}}+\frac{\lambda \varphi_{3}^{2}}{M_{3}^{2}}\right]-\frac{\partial^{3} V_{T}\left(\varphi_{3}, R_{3}\right)}{\partial \phi_{c}^{2} \partial R} \\
\delta \varepsilon_{0}= & \frac{(\xi-1 / 6) m^{2}}{32 \pi^{2}}\left[1-\ln \frac{m^{2}}{\mu^{2}}\right] \\
\delta \varepsilon_{1}= & -\frac{(\xi-1 / 6)^{2}}{32 \pi^{2}} \ln \frac{M_{5}^{2}}{\mu^{2}}-\frac{\partial^{2} V_{T}\left(\varphi_{5}, R_{5}\right)}{\partial R^{2}}
\end{aligned}
$$

where the fact that the topological contribution $V_{T}\left(\phi_{c}, R\right)$ to the effective potential is exponentially vanishing for $R \rightarrow 0$ has been used in writing the latter expressions. Moreover, we have set

$$
M_{q}^{2}=a^{2}\left(\varphi_{q}, R_{q}\right)=m^{2}+\left(\xi-\frac{1}{6}\right) R_{q}+\frac{\lambda \varphi_{q}^{2}}{2}
$$

The final expression for the renormalized one-loop effective potential does not depend on the arbitrary scale parameter $\mu$. We have

$$
\begin{aligned}
V_{r}^{(1)}\left(\phi_{c}, R\right)= & -\frac{m^{2} \varphi_{0}^{2}}{2}-\frac{\lambda \varphi_{0}^{4}}{24}+\frac{\lambda^{2} \phi_{c}^{4}}{256 \pi^{2}}\left[\left(\ln \frac{a^{2}}{M_{1}^{2}}-\frac{25}{6}\right)+\frac{4 m^{2}\left(m^{2}+M_{1}^{2}\right)}{3 M_{1}^{4}}\right] \\
& +\frac{\lambda \phi_{c}^{2}}{64 \pi^{2}}\left[\left(\xi-\frac{1}{6}\right) R\left(\ln \frac{a^{2}}{M_{3}^{2}}-\frac{3}{2}-\frac{\lambda \varphi_{3}^{2}}{M_{3}^{2}}\right)+m^{2}\left(\ln \frac{a^{2}}{m^{2}}-\frac{1}{2}\right)\right] \\
& +\frac{1}{64 \pi^{2}}\left[2 m^{2}\left(\xi-\frac{1}{6}\right) R\left(\ln \frac{a^{2}}{m^{2}}-\frac{1}{2}\right)+\left(\xi-\frac{1}{6}\right)^{2} R^{2}\left(\ln \frac{a^{2}}{M_{5}^{2}}-\frac{3}{2}\right)\right. \\
& +m^{4} \ln \frac{a^{2}}{M_{0}^{2}}-\lambda m^{2} \varphi_{0}^{2}\left(\ln \frac{M_{0}^{2}}{m^{2}}-\frac{1}{2}\right)
\end{aligned}
$$




$$
\begin{array}{r}
\left.-\frac{\lambda^{2} \varphi_{0}^{4}}{4}\left(\ln \frac{M_{0}^{2}}{M_{1}^{2}}-\frac{3}{2}-\frac{4\left(M_{1}^{2}-m^{2}\right)\left(2 M_{1}^{2}+m^{2}\right.}{3 M_{1}^{4}}\right)\right] \\
+V_{T}\left(\phi_{c}, R\right)+\frac{\partial^{4} V_{T}\left(\varphi_{1}, R_{1}\right)}{\partial \phi_{c}^{4}}\left(\frac{\phi_{c}^{4}-\varphi_{0}^{4}}{24}\right) \\
-\frac{\partial^{3} V_{T}\left(\varphi_{3}, R_{3}\right)}{\partial \phi_{c}^{2} \partial R} \frac{R \phi_{c}^{2}}{2}-\frac{\partial^{2} V_{T}\left(\varphi_{5}, R_{5}\right)}{\partial R^{2}} \frac{R^{2}}{2}
\end{array}
$$

Expression (4.8) notably simplifies in the special but important case of a conformal invariant scalar field. First of all we observe that the scalar curvature of a manifold of the kind $R \times H^{N} / \Gamma$ is just the same as one has for $H^{N}$. This means that $R=N(N-1) \kappa$ and

$$
a^{2}=m^{2}+\{\xi-(D-2) / 4(D-1)\} R+V_{o}^{\prime \prime}\left(\phi_{c}\right)
$$

Then we see that for a conformally coupled scalar field, $a^{2}=\lambda \phi_{c}^{2} / 2$ does not depend on the curvature. One easily gets

$$
\begin{aligned}
V_{r}^{(1)}\left(\phi_{c}, R\right)= & \frac{\lambda^{2} \phi_{c}^{4}}{256 \pi^{2}}\left[\ln \frac{\phi_{c}^{2}}{\varphi_{1}^{2}}-\frac{25}{6}\right]-\frac{\lambda^{2} \varphi_{0}^{4}}{256 \pi^{2}}\left[\ln \frac{\varphi_{0}^{2}}{\varphi_{1}^{2}}-\frac{25}{6}\right]-\frac{\lambda \varphi_{0}^{4}}{24}+V_{T}\left(\phi_{c}, R\right) \\
& +\frac{\partial^{4} V_{T}\left(\varphi_{1}, R_{1}\right)}{\partial \phi_{c}^{4}}\left(\frac{\phi_{c}^{4}-\varphi_{0}^{4}}{24}\right)-\frac{\partial^{3} V_{T}\left(\varphi_{3}, R_{3}\right)}{\partial \phi_{c}^{2} \partial R} \frac{R \phi_{c}^{2}}{2}
\end{aligned}
$$

which reduces to the potential of Coleman-Weinberg [18] in the flat space limit.

\section{The renormalization group equations for $\lambda \phi^{4}$}

In the present section we derive the renormalization group equations for the $\lambda \phi^{4}$ theory [31] on a 4-dimensional compact smooth manifold without boundary, the extension to manifolds with boundary being straightforward. The derivation is presented only for the sake of completeness and is valid only at one-loop level. For a more general discussion see for example [32] and references cited therein. 
It is convenient to regularize the one-loop effective action (2.4) by means of the zetafunction technique [33]. In this way the one-loop effective action is finite and reads

$$
\Gamma\left[\phi_{c}, g\right]=S_{c}\left[\phi_{c}, g\right]+\frac{1}{2} \zeta^{\prime}\left(0 ; A / \mu^{2}\right)=\int \mathcal{L} \sqrt{g} d^{4} x
$$

where $\zeta^{\prime}\left(s ; A / \mu^{2}\right)$ is the derivative of the zeta-function related to the small disturbance operator $A=L+\lambda \phi_{c}^{2} / 2$. The classical action is assumed to be of the form

$$
S_{c}\left[\phi_{c}, g\right]=\int\left[\frac{\phi_{c} L \phi_{c}}{2}+\frac{\lambda \phi_{c}^{4}}{24}\right] \sqrt{g} d^{4} x+\int\left[\Lambda+\varepsilon_{0} R+\frac{\varepsilon_{1} R^{2}}{2}+\varepsilon_{2} C^{2}\right] \sqrt{g} d^{4} x
$$

$C^{2}=C^{i j r s} C_{i j r s}$ being the square of the Weyl tensor. Note however that $C^{2}=0$ on the manifold under consideration and so such a term has been omitted in the previous section.

In order to see how the conformal anomaly enters into the game, we consider a conformal transformation $\tilde{g}_{i j}=\exp (2 \sigma) g_{i j}$ with $\sigma$ a constant (scaling). By the conformal transformation properties of the fields, one can easily check that $\tilde{S}_{c}(\alpha)=S_{c}(\tilde{\alpha})$, where as before $\alpha$ represents the collection of all coupling constants and $\tilde{\alpha}$ are all equal to $\alpha$ apart from $\Lambda, m^{2}$, and $\varepsilon_{0}$. For these we have

$$
\tilde{\Lambda}=\Lambda e^{4 \sigma} ; \quad \tilde{m}^{2}=m^{2} e^{2 \sigma} ; \quad \tilde{\varepsilon}_{0}=\varepsilon_{0} e^{2 \sigma} .
$$

In the same way, for the eigenvalues $\tilde{\mu}_{n}(\alpha)$ of the small disturbance operator $\tilde{A}(\alpha)$ we have $\tilde{\mu}_{n}(\alpha)=e^{-2 \sigma} \mu_{n}(\tilde{\alpha})$. From this transformation rule for the eigenvalues, we immediately get the transformations for $\zeta\left(s ; A / \mu^{2}\right)$ and $\zeta^{\prime}\left(s ; A / \mu^{2}\right)$. They read

$$
\begin{aligned}
\zeta\left(s ; \tilde{A} / \mu^{2}\right) & =e^{2 s \sigma} \zeta\left(s ; A(\tilde{\alpha}) / \mu^{2}\right) \\
\zeta^{\prime}\left(s ; \tilde{A} / \mu^{2}\right) & =e^{2 s \sigma}\left[\zeta^{\prime}\left(s ; A(\tilde{\alpha}) / \mu^{2}\right)+2 \sigma \zeta\left(s ; A\left(\tilde{\alpha} / \mu^{2}\right)\right]\right.
\end{aligned}
$$


and finally

$$
\tilde{\Gamma}(\alpha)=\Gamma(\tilde{\alpha})-\sigma \zeta(0 ; A(\tilde{\alpha}))
$$

It is well known that $\zeta(0 ; A)$ is related to the Seeley-DeWitt coefficient $a_{D / 2}(x ; A)$ by means of the equation

$$
\zeta(0 ; A)=\frac{1}{(4 \pi)^{D / 2}} \int a_{D / 2}(x ; A) \sqrt{g} d^{D} x
$$

(see the Appendix B for details on zeta-function and heat kernel expansion). What is relevant for our case is $a_{2}(x ; A(\tilde{\alpha}))$ which is known to be [34, 35]

$$
\begin{aligned}
a_{2}(x ; A(\tilde{\alpha}))= & -\frac{\lambda \triangle \phi_{c}^{2}}{12}+\frac{\lambda \tilde{m}^{2} \phi_{c}^{2}}{2}+\frac{\lambda(\xi-1 / 6) R \phi_{c}^{2}}{2}+\frac{\lambda^{2} \phi_{c}^{4}}{8}+\frac{\tilde{m}^{4}}{2} \\
& +\tilde{m}^{2}\left(\xi-\frac{1}{6}\right) R+\frac{(\xi-1 / 6)^{2} R^{2}}{2}+\frac{C^{2}}{120}-\frac{G}{360}+\frac{(5-\xi) \triangle R}{6}
\end{aligned}
$$

where $G=R^{i j r s} R_{i j r s}-4 R^{i j} R_{i j}+R^{2}$ is the Gauss-Bonnet density, which is a total divergence in 4 dimensions. Integrating $a_{2}$ on the manifold and disregarding all total divergences one finally gets

$$
\tilde{\Gamma}(\alpha)=S_{c}(\tilde{\alpha}(\sigma))+\frac{1}{2} \zeta^{\prime}(0 ; \tilde{A})=\Gamma(\tilde{\alpha}(\sigma))+o\left(\hbar^{2}\right)
$$

The new parameters $\alpha(\sigma)$ are related to the old ones $\alpha=\alpha(0)$ by

$$
\begin{array}{lll}
\Lambda(\sigma)-\Lambda=\frac{m^{4}}{2} \hat{\sigma} ; & \lambda(\sigma)-\lambda=3 \lambda^{2} \hat{\sigma} ; & m^{2}(\sigma)-m^{2}=\lambda m^{2} \hat{\sigma} ; \\
\xi(\sigma)-\xi=\left(\xi-\frac{1}{6}\right) \lambda \hat{\sigma} ; & \varepsilon_{0}(\sigma)-\varepsilon_{0}=\left(\xi-\frac{1}{6}\right) m^{2} \hat{\sigma} ; & \varepsilon_{1}(\sigma)-\varepsilon_{1}=\left(\xi-\frac{1}{6}\right)^{2} \hat{\sigma} ; \\
\varepsilon_{2}(\sigma)-\varepsilon_{2}=\frac{1}{30} \hat{\sigma} . &
\end{array}
$$

Here we have set $\hat{\sigma}=-\sigma / 16 \pi^{2}$. With the substitution $\sigma \rightarrow-2 \tau$, the latter equations have been given for example in refs. [36, 29]. These important formulae tell us that all 
parameters (coupling constants) develop as a result of quantum effects a scale dependence even if classically they are dimensionless parameters. This means that in the quantum case we have to define the coupling constants at some particular scale. Differentiating eq. (5.9) with respect to $\sigma$, one immediately gets the renormalization group equations [36, 29].

\section{Symmetry breaking and topological mass genera-}

\section{tion}

Let us now concentrate on the physical implications of the one-loop effective potential, eq. (4.8). In order to analyze the phase transition of the considered system, let us specialyze to different cases and to several limits. For this discussion, we will consider $R_{1}=R_{3}=R_{5}=0$. In this way, for $q=1,3,5$ we have $M_{q}^{2}=m^{2}+\lambda \varphi_{q}^{2} / 2$.

Let us first concentrate on the regime $m^{2}+\xi R>0$. Then $\phi_{c}=0$ is a minimum of the classical potential and the expansion of the quantum corrections around the classical minimum reads

$$
\begin{aligned}
V_{r}=\Lambda_{e f f} & +\frac{\left(m^{2}+\xi R+m_{T}^{2}\right) \phi_{c}^{2}}{2}+\frac{\lambda \phi_{c}^{2}}{64 \pi^{2}}\left[m^{2} \ln \frac{m^{2}+(\xi-1 / 6) R}{m^{2}}\right. \\
& \left.+R\left(\xi-\frac{1}{6}\right)\left(\ln \frac{m^{2}+(\xi-1 / 6) R}{M_{3}^{2}}-\frac{\lambda \varphi_{3}^{2}}{M_{3}^{2}}-1\right)\right]+\mathcal{O}\left(\phi_{c}^{4}\right)
\end{aligned}
$$

where $\Lambda_{\text {eff }}$ (the effective cosmological constant) represents a complicated expression not depending on the background field $\phi_{c}$ and $m_{T}^{2}$ is the topological contribution to the mass of the field, which reads

$$
m_{T}^{2}=\frac{\sqrt{3} \lambda|R|^{-1 / 2}}{4 \pi V(\mathcal{F})} \int_{1}^{\infty} d u\left(u^{2}-1\right)^{-\frac{1}{2}} \frac{Z^{\prime}}{Z}\left(1+u \sqrt{6 m^{2}|R|^{-1}+1-6 \xi}\right)
$$


Let us first restrict our attention to the small curvature limit. Then, as mentioned, the topological part is negligible and the leading orders only including the linear curvature terms are easily obtained from eq. 6.1). We obtain

$$
V_{r}=\Lambda_{e f f}+\frac{\left(m^{2}+\xi R\right) \phi_{c}^{2}}{2}-\frac{\lambda \phi_{c}^{2}}{64 \pi^{2}} R\left(\xi-\frac{1}{6}\right)\left[\frac{\lambda \varphi_{3}^{2}}{M_{3}^{2}}-\ln \frac{m^{2}}{M_{3}^{2}}\right]
$$

This result in fact is true for any smooth manifold, when the constant curvature $R$ in the considered manifold is replaced by the respective scalar curvature of the manifold. The recent contribution of ref. 30 on the Coleman-Weinberg symmetry breaking in a Bianchi type-I universe is another example of the general structure, which may be proven by the use of heat-kernel techniques. Due to $R<0$ in our example, for $\xi<\frac{1}{6}$ the one loop term will help to break symmetry, whereas for $\xi>\frac{1}{6}$ the quantum contribution acts as a positive mass and helps to restore symmetry. The quantum corrections should be compared to the classical terms which in general dominate, because the one loop terms are suppressed by the square of the Planck mass. But if $\xi$ is small enough the one loop term will be the most important and will then help to break symmetry.

Let us now consider the opposite limit, that is $|R| \rightarrow \infty$ with the requirement $\xi<0$. In that range the leading order of the topological part reads

$$
m_{T}^{2}=\frac{\sqrt{3} \lambda|R|^{-1 / 2}}{4 \pi V(\mathcal{F})} \int_{1}^{\infty} d u\left(u^{2}-1\right)^{-\frac{1}{2}} \frac{Z^{\prime}}{Z}(1+u \sqrt{1-6 \xi})+\mathcal{O}\left(R^{0}\right)
$$

It is linear in the scalar curvature $R$ because $V(\mathcal{F}) \sim|R|^{-\frac{3}{2}}$. The sign of the contribution depends on the choice of the characters $\chi\left(P^{3}(\gamma)\right)\left(S_{3}\left(n ; l_{\gamma}\right)\right.$ is always positive). However, for trivial character $\chi=1$ we can say, that the contribution helps to restore the symmetry, whereas for nontrivial character it may also serve as a symmetry breaking mechanism. 
So, if the symmetry is broken at small curvature, for $\chi=1$ a symmetry restoration at some critical curvature $R_{c}$, depending strongly on the non trivial topology, will take place.

Let us now say some words on the case $m^{2}+\xi R<0$, which includes for example the conformal invariant case. As we mentioned in section 4 the classical potential has two minima at $\pm\left(-6\left[m^{2}+\xi R\right] / \lambda\right)^{\frac{1}{2}}$. So even for $\xi>0$ due to the negative curvature in the given space-time the classical starting point is a theory with a broken symmetry. As is seen in the previous discussion, in order to analyze the influence of the quantum corrections on the symmetry of the classical potential a knowledge of the function $Z^{\prime}(s) / Z(s)$ for values $\operatorname{Re} s<2$ is required. Unfortunately, the defining product for the Selberg zeta-function is only absolutely convergent in the range Re $s>2$ [37], so an analytic continuation of this product to $\operatorname{Re} s<2$ is needed. Although the analytic continuation exists ([37, Theorem 4.1, p.93), using known properties of this continuation it is not possible to determine some definite sign of expressions like equation (6.2). So even for trivial character $\chi=1$ it is not possible to say, whether the topology helps to restore the symmetry or not.

\section{Conclusions}

In new inflationary models, the effective cosmological constant is obtained from an effective potential, which includes quantum corrections to the classical potential of a scalar field [18]. This potential is usually calculated in Minkowski space-time, whereas to be fully consistent, the effective potential must be calculated for more general space-times. For that reason an intensive research has been dedicated to the analysis of the one-loop effective potential of a self-interacting scalar field in curved space-time. Especially to be 
mentioned are the considerations on the torus [6, 7, 9, 12, 13], which, however, do not include nonvanishing curvature, and the quasi-local approximation scheme developed in [29], which, however, fails to incorporate global properties of the space-time. To overcome this deficiency, we were naturally led to the given considerations on the space-time manifold $R \times H^{3} / \Gamma$. Apart from its physical relevance [15], this manifold combines nonvanishing curvature with highly nontrivial topology, still permitting the exact calculation of the one-loop effective potential (4.8) by the use of the Selberg trace formula (3.1). So, at least for $m^{2}+\xi R>0$ and trivial line bundles, $\chi=1$, we were able to determine explicitly the influence of the topology, namely the tendency to restore the symmetry. Furthermore, this contribution being exponentially damped for small curvature, we saw,

in that regime, that for $\xi<\frac{1}{6}$ the quantum corrections to the classical potential can help to break symmetry.

\section{Acknowledgments}

We would like to thank A. A. Bytsenko, S. D. Odintsov and L. Vanzo for helpful discussions and suggestions. K. Kirsten is grateful to Prof. M. Toller, Prof. R. Ferrari and Prof. S. Stringari for the hospitality in the Theoretical Group of the Department of Physics of the University of Trento.

\section{A Appendix: admissible regularizations}

For the sake of completeness we give in this appendix a list of admissible regularization functions, which are often used in the literature (see also ref. [17]). We limit our analysis to $D=4$. 
Let us start with

$$
\varrho_{1}(\varepsilon, t)=\frac{d}{d \varepsilon} \frac{t^{\varepsilon}}{\Gamma(\varepsilon)}=\frac{t^{\varepsilon}}{\Gamma(\varepsilon)}\{\ln t-\psi(\varepsilon)\}
$$

where $\psi(\varepsilon)$ is the logarithmic derivative of $\Gamma(\varepsilon)$. This is the well known zeta-function regularization [33. All requirements are satified. The related $B_{e}(\varepsilon, y)$ and $V(\varepsilon, f)$, say $B_{1}(\varepsilon, y)$ and $V_{1}(\varepsilon, f)$ read

$$
\begin{aligned}
B_{1}(\varepsilon, y) & =y^{-\varepsilon} \ln y=\ln y+\mathcal{O}(\varepsilon) \\
V_{1}(\varepsilon) & =-\frac{\mu^{4} y^{2}}{32 \pi^{2}} \frac{d}{d \varepsilon} \frac{\Gamma(\varepsilon-2) y^{-\varepsilon}}{\Gamma(\varepsilon)}=\frac{\mu^{4} y^{2-\varepsilon}}{64 \pi^{2}} \frac{\Gamma(\varepsilon-2)}{\Gamma(\varepsilon)}[\ln y-\psi(\varepsilon-2)+\psi(\varepsilon)] \\
& =\frac{f^{4}}{64 \pi^{2}}\left[\ln \frac{f^{2}}{\mu^{2}}-\frac{3}{2}\right]+\mathcal{O}(\varepsilon)
\end{aligned}
$$

We see that the effective potential is finite, the divergent terms being removed by the particular structure of $\varrho_{1}$.

The next regularization we shall consider is closely related to the above one, and in some sense is associated with the familiar dimensional regularization in momentum space. It is defined by 38

$$
\varrho_{2}(\varepsilon, t)=t^{\varepsilon}
$$

From this,

$$
\begin{aligned}
B_{2}(\varepsilon, y) & =-y^{-\varepsilon} \Gamma(\varepsilon)=\ln y+\gamma-\frac{1}{\varepsilon}+\mathcal{O}(\varepsilon) \\
V_{2}(\varepsilon) & =-\frac{\mu^{4} y^{2-\varepsilon}}{32 \pi^{2}} \Gamma(\varepsilon-2) \\
& =\frac{f^{4}}{64 \pi^{2}}\left[\ln \frac{f^{2}}{\mu^{2}}-\frac{3}{2}+\gamma\right]-\frac{f^{4}}{64 \pi^{2} \varepsilon}+\mathcal{O}(\varepsilon)
\end{aligned}
$$


easily follow, again in agreement with the general result. Within this regularization, only one divergent term is present.

Another often used regularization is the ultraviolet cut-off regularization, defined by

$$
\varrho_{3}(\varepsilon, t)=\theta(t-\varepsilon)
$$

In this case we have

$$
\begin{aligned}
B_{3}(\varepsilon, y) & =-\Gamma(0, y \varepsilon)=\ln y+\gamma+\ln \varepsilon+\mathcal{O}(\varepsilon) \\
V_{3}(\varepsilon) & =-\frac{\mu^{4} y^{2} \Gamma(-2, \varepsilon y)}{32 \pi^{2}} \\
& =\frac{f^{4}}{64 \pi^{2}}\left[\ln \frac{f^{2}}{\mu^{2}}-\frac{3}{2}+\gamma\right]-\frac{\mu^{4}}{64 \pi^{2} \varepsilon^{2}}+\frac{\mu^{2} f^{2}}{32 \pi^{2} \varepsilon}+\frac{f^{4}}{64 \pi^{2}} \ln \varepsilon+\mathcal{O}(\varepsilon)
\end{aligned}
$$

Here we have three divergent terms. From these examples, it is obvious that they depend on the regularization function.

The fourth regularization reads

$$
\varrho_{4}(\varepsilon, t)=e^{-\varepsilon / 4 t}
$$

We have

$$
\begin{aligned}
B_{4}(\varepsilon, y) & =-2 \mathbf{K}_{0}(\sqrt{\varepsilon y})=\ln y+2 \gamma-2 \ln 2+\ln \varepsilon+\mathcal{O}(\varepsilon) \\
V_{4}(\varepsilon) & =-\frac{\mu^{4} y}{4 \varepsilon \pi^{2}} \mathbf{K}_{2}(\sqrt{\varepsilon y}) \\
& =\frac{f^{4}}{64 \pi^{2}}\left[\ln \frac{f^{2}}{\mu^{2}}-\frac{3}{2}+2 \gamma-2 \ln 2\right]-\frac{\mu^{4}}{2 \pi^{2} \varepsilon^{2}}+\frac{\mu^{2} f^{2}}{8 \pi^{2} \varepsilon}+\frac{f^{4}}{64 \pi^{2}} \ln \varepsilon+\mathcal{O}(\varepsilon)
\end{aligned}
$$

$\mathbf{K}_{\nu}$ being the Mc Donald functions. 
The last regularization we would like to consider is presented as an example of the freedom one has. It is similar to a Pauli-Villars type and it is defined by ( $\alpha$ being an arbitrary positive constant)

$$
\varrho_{5}(\varepsilon, t)=\left(1-e^{-\alpha t / \varepsilon}\right)^{3}
$$

the power 3 being related to the fact that we are working in four dimensions. This is a general feature of the Pauli-Villars regularization. We have

$$
\begin{gathered}
B_{5}(\varepsilon, y)=\ln \frac{\varepsilon y}{\varepsilon y+3 \alpha}+3 \ln \frac{\varepsilon y+2 \alpha}{\varepsilon y+\alpha}=\ln y+\ln \frac{8}{3 \alpha}+\ln \varepsilon+\mathcal{O}(\varepsilon) \\
\begin{aligned}
V_{5}(\varepsilon)= & \frac{\mu^{4} y^{2}}{64 \pi^{2}}\left\{\left[\ln \frac{\varepsilon y}{\alpha}-\frac{3}{2}\right]-3\left(1+\frac{\alpha}{\varepsilon y}\right)^{2}\left[\ln \left(1+\frac{\varepsilon y}{\alpha}\right)-\frac{3}{2}\right]\right. \\
& \left.+3\left(1+\frac{2 \alpha}{\varepsilon y}\right)^{2}\left[\ln \left(2+\frac{\varepsilon y}{\alpha}\right)-\frac{3}{2}\right]-\left(1+\frac{3 \alpha}{\varepsilon y}\right)^{2}\left[\ln \left(3+\frac{\varepsilon y}{\alpha}\right)-\frac{3}{2}\right]\right\} \\
=\frac{f^{4}}{64 \pi^{2}}\left[\ln \frac{f^{2}}{\mu^{2}}-\frac{3}{2}+\ln \frac{8 \alpha}{3}\right]+\frac{3 \alpha^{2} \ln (16 / 27) \mu^{4}}{64 \pi^{2} \varepsilon^{2}} & +\frac{3 \alpha \ln (16 / 9) \mu^{2} f^{2}}{64 \pi^{2} \varepsilon}+\frac{f^{4}}{64 \pi^{2}} \ln \varepsilon+\mathcal{O}(\varepsilon)
\end{aligned}
\end{gathered}
$$

With this example we conclude the list of possible regularization functions.

\section{B Appendix: heat kernel expansion and zeta-function}

For convenience of the reader, here we just report on some results concerning heat kernel expansion and zeta function. To start with we consider a second order elliptic differential operator $A=-\triangle+X(x)$ defined on a smooth $D$-dimensional Riemannian manifold without boundary $(X(x)$ is a scalar function). The results we are going to discuss have been quite recently given also for Riemannian manifolds with boundary [39, 40, 41, 42] 
and also in the presence of torsion [43, 44]. The kernel of the operator $\exp (-t A)$, which satisfies a heat type equation, has an asymptotic expansion, valid for small $t$, given by

$$
K_{t}(x, x ; A) \sim \frac{1}{(4 \pi t)^{N / 2}} \sum_{n=0}^{\infty} a_{n}(x ; A) t^{n}
$$

$a_{n}(x ; A)$ being the spectral (Minakshisundaram, Seeley, DeWitt, Gilkey) coefficients, which are invariant quantities built up with curvature and their derivatives. Some of such coefficients are computed by many authors (see for example refs. [39]). In particular we have (of course $a_{0}(x ; A)=1$ )

$$
\begin{aligned}
& a_{1}(x ; A)=\frac{R}{6}-X \\
& a_{2}(x ; A)=\frac{1}{2} a_{1}^{2}+\frac{1}{6} \triangle a_{1}+\frac{1}{180}\left[\triangle R+R^{i j r s} R_{i j r s}-R^{i j} R_{i j}\right]
\end{aligned}
$$

Sometimes it may be convenient to factorize the exponential $\exp \left(t a_{1}\right)$ and consider an expansion very closely related to eq. (B.1), that is [45]

$$
K_{t}(x, x ; A) \sim \frac{e^{-t(X-R / 6)}}{(4 \pi t)^{N / 2}} \sum_{n=0}^{\infty} b_{n}(x ; A) t^{n}
$$

with $b_{0}=1, b_{1}=0$ and more generally

$$
\begin{aligned}
& a_{n}(x ; A)=\sum_{l=0}^{n} \frac{b_{n-l} a_{1}^{l}}{l !} \\
& b_{n}(x ; A)=\sum_{l=0}^{n} \frac{(-1)^{l} a_{n-l} a_{1}^{l}}{l !}
\end{aligned}
$$

In this way, all coefficients $b_{n}$ do not depend on $a_{1}$ [45].

The connection between heat kernel and zeta-function is realized by means of the Mellin representation

$$
\zeta(s ; A)=\frac{1}{\Gamma(s)} \int_{0}^{\infty} t^{s-1} \operatorname{Tr} e^{-t A} d t
$$


Using expansion (B.1) in this latter expression, we can get the meromorphic structure of $\zeta(s ; A)$ as given by Seeley in ref. 46]. If we suppose $-a_{1}$ to be a positive function, say $a^{2}$, we can obtain the zeta-function as a power series of the kind

$$
\zeta\left(s ; A / \mu^{2}\right) \sim \sum_{n \geq 0} \frac{\mu^{2 s} \Gamma(s+n-D / 2)}{(4 \pi)^{D / 2} \Gamma(s)} \int_{\mathcal{M}} b_{n} a^{-2(s+n-D / 2)} \sqrt{g} d^{D} x
$$

from which eq. (5.6) follows and for $D=4$ one obtains furthermore

$$
\zeta^{\prime}\left(0 ; A / \mu^{2}\right) \sim \frac{1}{16 \pi^{2}} \int_{\mathcal{M}}\left[\frac{3}{4} a^{4}-a_{2} \ln \frac{a^{2}}{\mu^{2}}+\sum_{n \geq 1} \frac{(n-1) ! b_{n+2}}{a^{2 n}}\right] \sqrt{g} d^{4} x
$$

concluding the summary of useful results.

\section{References}

[1] N.D. Birrell and P.C.W. Davies. Quantum fields on curved space. Cambridge University Press, Cambridge, England, (1982).

[2] R.H. Brandenberger. Rev. Mod. Phys., 57, 1, (1985).

[3] R. Jackiw. Phys. Rev. D, 9, 1686, (1974).

[4] D.J. Toms. Phys. Rev., 26, 2713, (1982).

[5] L.Ford and T. Yoshimura. Phys. Lett. A, 70, 89, (1979).

[6] L.Ford . Phys. Rev. D, 21, 933, (1980).

[7] D.J. Toms. Phys. Rev. D, 21, 2805, (1980).

[8] D.J. Toms. Ann. Phys., 129, 334, (1980).

[9] G. Denardo and E. Spallucci. Nuovo Cimento A, 58, 243, (1980). 
[10] G. Denardo and E. Spallucci. Nucl. Phys. B, 169, 514, (1980).

[11] G. Kennedy. Phys. Rev. D, 23, 2884, (1981).

[12] A. Actor. Class. Quantum Grav., 7, 1463, (1990).

[13] E. Elizalde and A. Romeo. Phys. Lett. B, 244, 387, (1990).

[14] P. Chang and J.S. Dowker. Vacuum energy on orbifold factors of spheres. Technical Report, Preprint, Manchester University, (1992).

[15] G.F.R. Ellis. Gen. Rel. Grav., 2, 7, (1971).

[16] W.P. Thurston. Bull. Amer. Math. Soc., 6, 357, (1982).

[17] R.D. Ball. Phys. Rep., 182, 1, (1989).

[18] S. Coleman and E. Weinberg. Phys. Rev., 7, 1888, (1973).

[19] J. Iliopoulos, C. Itzykson and A. Martin. Rev. Mod. Phys., 47, 165, (1975).

[20] J. Elstrodt, F. Grunewald and J. Mennicke. Math. Ann., 277, 655, (1987).

[21] A. Selberg. J. Indian Math Soc., 20, 47, (1956).

[22] I. Chavel. Eigenvalues in Riemannian geometry. Academic Press, Inc. U.S.A., (1984).

[23] D.J. Toms. Phys. Rev. D, 21, 928, (1979).

[24] N.D. Birrell and L. Ford. Phys. Rev. D, 22, 330, (1980).

[25] B.S. Kay. Phys. Rev. D, 20, 3052, (1979).

[26] I.S. Gradshteyn and I.M Ryzhik. Table of integrals, series and products. Academic press, Inc., New York, (1980). 
[27] A.A. Bytsenko and S. Zerbini. Class. Quantum Grav., 9, 1365, (1992).

[28] R. Utiyama and B. DeWitt. J. Math. Phys., 3, 608, (1962).

[29] B.L. Hu and D.J. O'Connors . Phys. Rev., 30, 743, (1984).

[30] A.L. Berkin. Phys. Rev., 46, 1551, (1992).

[31] B.L. Nelson and P. Panangaden. Phys. Rev., 25, 1019, (1982).

[32] I.L. Buchbinder, S.D. Odintsov and I.L. Shapiro. Effective Action in Quantum Gravity. A. Hilger, Bristol, (1992).

[33] S.W. Hawking. Comm. Math. Phys., 55, 133, (1977).

[34] B.S. DeWitt. The dynamical theory of groups and fields. Gordon and Breach, New York, (1965).

[35] P.B. Gilkey. J. Diff. Geometry, 10, 601, (1975).

[36] D.J. O'Connors, B.L. Hu and T.C. Shen . Phys. Lett. B, 130, 31, (1983).

[37] A.B. Venkov. Russian Math. Surveys, 34, 79, (1979).

[38] J.S. Dowker and R. Critchley. Phys. Rev. D, 13, 3224, (1976).

[39] T.P. Branson and P.B.Gilkey. Comm. Part. Diff. Eqs., 15, 245, (1990).

[40] G. Cognola, L. Vanzo and S. Zerbini. Phys. Lett. B, 241, 381, (1990).

[41] D.M. McAvity and H. Osborn. Class. Quantum Grav., 8, 603, (1991).

[42] A. Dettki and A. Wipf. Nucl. Phys. B, 377, 252, (1992).

[43] Yu.N. Obukhov. Phys. Lett. B, 108, 308, (1982). 
[44] G. Cognola and S. Zerbini. Phys. Lett. B, 214, 70, (1988).

[45] I. Jack and L. Parker. Phys. Rev. D, 31, 2439, (1985).

[46] R.T. Seeley. Am. Math. Soc. Prog. Pure Math., 10, 172, (1967). 